\title{
Change in disparities of dietary quality score (FSAm-NPS) in adolescents and young adults between 2004 and 2014 in Belgium
}

\author{
Lucille Desbouys $^{1}$, Manon Rouche ${ }^{1}$, Karin De Ridder ${ }^{2}$ and Katia Castetbon ${ }^{1}$ \\ ${ }^{1}$ Université libre de Bruxelles, Ecole de santé publique, Brussels, Belgium and \\ ${ }^{2}$ Sciensano, Department of Epidemiology and Public Health and Surveillance, Brussels, Belgium
}

\section{Abstract}

Introduction

The transition from adolescence toward adulthood is a critical period regarding changes in dietary behaviours. Moreover at these ages, socio-economic and regional disparities in food group consumption are observed. The aim of our study was to determine how the nutritional quality of diet, measured by the modified Nutrient Profiling System of the British Food Standards Agency (FSAm-NPS), evolved between 2004 and 2014, according to socio-economic and regional characteristics of adolescents and young adults living in Belgium.

\section{Material and methods}

Two non-consecutive 24-hour dietary recalls were carried out in two nationally-representative samples of 15-to-39-year-old respondents included in the Belgian Food Consumption Surveys in $2004(n=1,186)$ and $2014(n=952)$. Weighting factor (according to age, gender, day of recall, season and province) and sample design were considered. The weighted mean individual FSAm-NPS was computed from all foods and beverages consumed and converted into a scale from 0 (less favourable diet) to 100 (more favourable diet). Slope (SII) and Relative (RII) Index of Inequality were compared between 2004 and 2014 in three age groups (15-18 y, 19-25 y and 26-39 y), adjusting for gender, energy intake, and other socio-economic and regional characteristics.

\section{Results}

In the three age groups, the weighted mean FSAm-NPS significantly increased between 2004 and 2014 (2004: 55.2 (SEM: 0.2) vs. 2014: $57.3(0.5), \mathrm{p}<0.001$ in 15-18-year-olds; 54.9 (0.6) vs. $58.0(0.4)$, p < 0.001 in 19-25-year-olds; $56.9(0.3)$ vs $58.3(0.3)$, $p<0.01$ in 26-39-year-olds). While a significant FSAm-NPS gradient was observed according to household education among 15-18- and 26-39-year-olds in 2004 (adjusted SII: 2.56 (95\% CI: 1.08-4.04) and 2.73 (0.34-5.12), respectively; RII: 1.05 (1.02-1.08) and 1.05 (1.01-1.09)), no significant difference was found in 2014. Conversely, no significant score disparity was observed according to household type in 2004, but disparities appeared in 2014: index of inequality were significant among subjects aged 19-25 y (SII: 3.89 (0.62-7.17); RII: 1.07 (1.01-1.13)) and 26-39 y (SII: 2.74 (0.31-5.17); RII: 1.05 (1.01-1.09)), the FSAm-NPS being more favourable for those living in two-parent families. The FSAm-NPS was generally higher for subjects living in Flanders than those in Wallonia (significant SII and RII only among 26-39-year-olds), the magnitude of regional disparities remaining stable over time.

\section{Discussion}

Overall FSAm-NPS improved during this 10-year period, but differentially according to family structure and household education. However, statistical power was weakened by the limited sample size. Additional investigations of changes in food group consumption disparities will complement our interpretations.

\section{Conflict of Interest}

There is no confict of interest. 